\title{
Effect of the tongue-in-groove technique on the smile form*
}

\author{
Alireza Mohebbi, Fatemeh Dehghani Firouzabadi, Mohammad Dehghani \\ Firouzabadi, Maryam Roomiani
}

ENT and Head \& Neck Research center and department, The five senses Institute, Iran University of Medical Sciences, Tehran, Iran
Rhinology 58: 6, 626 - 628, 2020

https://doi.org/10.4193/Rhin20.246

*Received for publication:

May 19, 2020

Accepted: July 20, 2020

\section{To the Editor:}

There are different qualitative and quantitative methods used to evaluate the smiling form, including the length and angles of the side view of the face ${ }^{(1)}$. Among the clinical factors used to estimate the esthetics of the smile, the Maxillary Incisor Show (MIS), is defined as "the vertical distance between the caudal upper lip in the midline and at the caudal-most aspect of the maxillary central incisors" (Figure $2{ }^{2}{ }^{(2)}$. Rhinoplasty can affect the smiling form and the surgeon needs to be aware of this important aspect of the procedure. It is important to evaluate both the quantitative and qualitative effects of rhinoplasty procedures such as the tongue-in-groove (TIG) maneuver on all aspects of beauty. This study is the first to focus on the effect of the TIG maneuver on the MIS length, which plays a key role in the quantitative measurement of the smile form. TIG seems to be a popular maneuver with a satisfaction rate of $97 \%{ }^{(3)}$. The technique is especially useful in correcting over-exposed columella and protruded nasal tip ${ }^{(4)}$. Patients who have a hanging columella resulting from a long caudal septum will benefit from this technique. Fixing the medial crura to the caudal septum will usually produce slight extension and lengthening of the upper lip. This is beneficial in patients with a very short upper lip ${ }^{(5)}$. The technique was previously used to correct the distance between the nasal septum and the lips ${ }^{(6)}$. Our senior surgeon performed this technique for ninety adult patients ( $84.5 \%$ female) who attended for septorhinoplasty after assessing them for exclusion criteria (i.e., patients with any history of connective tissue or neuromuscular disorders, upper jaw surgery, patients with facial lesions, history of trauma and revision cases) to evaluate the smile form. He elevated the flap of the septum anteriorly on both sides and detached the medial crura and posterior set back and sutured it to the caudal end of the septum with a PDS 5.0 suture passing from the mucosa (mucosal TIG) if the septum had the appropriate stability and length. The senior surgeon could determine the fixation spot based on the rotation and the projection in each case. An end-to-end or end-to-site septal extension graft was carried out if the stability or the length of the septum was inappropriate or where there was a septal caudal deviation (Figure 1). The distance from the suture entry to the medial crus and its caudal edge was the same on both sides. In all cases, the depressor muscle was only released but not cut in any patient. Standard full-face photos of the patients were taken in the maximum smile position with constant lens, light, and distance pre- and one-year post-operative, to rate the match between the nasolabial folds and the photographs. The distance from the upper lip to the tip of the incisors teeth was measured as the MIS by the ImageJ software. Finally, the effect of the TIG technique on the MIS length was evaluated quantitatively. Our findings showed that the mean MIS lengths of the patients were 5.88 ( \pm 1.59$) \mathrm{mm}$ before and $6.64( \pm 1.64) \mathrm{mm}$ after surgery. Nine patients had a shorter MIS post-surgery, 23 of them did not experience any change in their MIS length. However, the rest had longer MIS lengths from 1 to $3 \mathrm{~mm}$ (Appendix 1). Overall, the length of the MIS was increased in all but 9 (10\%) of our patients, with a mean of $0.77 \mathrm{~mm}$ ( $p$-value $<0.001$ ) one year post-operatively. However, the MIS change was significantly related to the initial MIS length ( $p$-value $=0.016$ ). The histogram of these changes is shown in Appendix 1 and representative patients are shown in Figure 2 . The MIS change was larger in patients with an initial MIS $\leq 4 \mathrm{~mm}$ and smaller in patients with an initial MIS $\geq 9 \mathrm{~mm}$. MIS changes in each subgroup are shown in Figure 2. However, the subgroup analysis showed that the MIS change was closely related to the initial MIS and the change was not significant in those with an initial MIS longer than $9 \mathrm{~mm}$. Although previous studies addressed the effect of TIG maneuver on upper tip projection ${ }^{(7)}$ as well as the association between the underlying skeletal, dental and soft tissue components and 


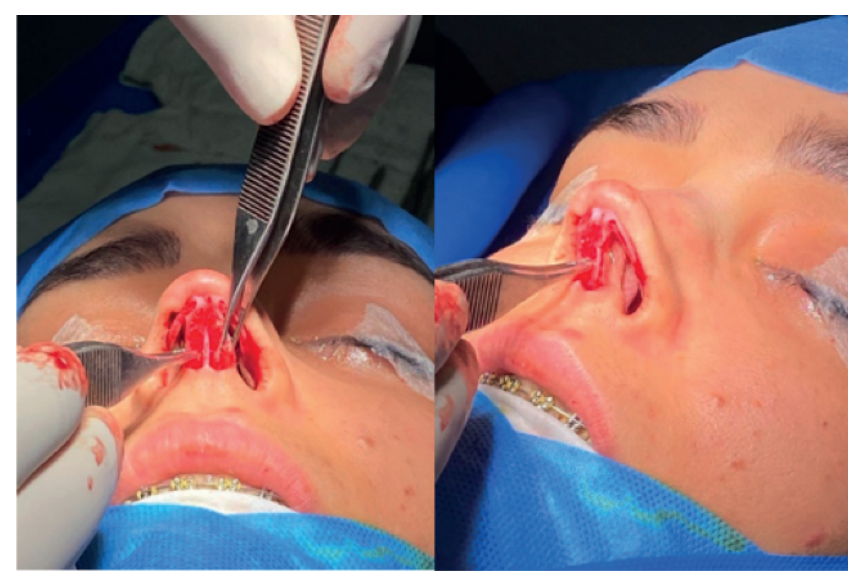

Figure 1. The mucosal tongue-in-groove technique.

the MIS, none of these studies focused on the effect of the TIG maneuver on the MIS length. For instance, Kalantar-Hormozi et al. ${ }^{\left({ }^{8}\right)}$ measured five smile parameters after two different rhinoplasty techniques such as resection (similar to the technique used by McCarthy and Wood-Smith et al. ${ }^{(9)}$ ) or transposition of the depressor septi nasi muscle (similar to the technique used by Rochrich et al. $\left.{ }^{(10)}\right)$. They found that although each technique significantly decreased the effect of smiling on some parameters, neither of them differed significantly in the amount that decreasing the effects of smiling had on all five parameters. Also, Hongyang et al. ${ }^{(2)}$ showed that rhinoplasty maneuvers can cause descent of the upper lip and decreased MIS when smiling. However, this study is the first report to quantitatively assess the effect of the mucosal TIG maneuver on a wide range of initial MIS lengths. From this study of patients with a gummy smile, it seems better not to do the mucosal TIG technique or perform this technique along with upper lip release surgery to improve patient satisfaction. We have had some cases with no change or even a decreased MIS in our patients that may be due to the spot of fixation and angle of rotation in the TIG maneuver. Caution is required in cases with more gingival show and shorter lip before surgery. Although release of the depressor septi nasi is done in all cases, we think that it is more important and noticeable in cases with more gingival show and a shorter lip before surgery.

In brief, our findings determined that the mucosal TIG technique can increase the MIS length, mostly in patients with shorter initial lengths. More comprehensive studies are needed to evaluate the effects of rhinoplasty techniques on semi-quantitative and quantitative measures of beauty and the smile form.

\section{Conflict of interest}

The authors declare that they have no competing interest.

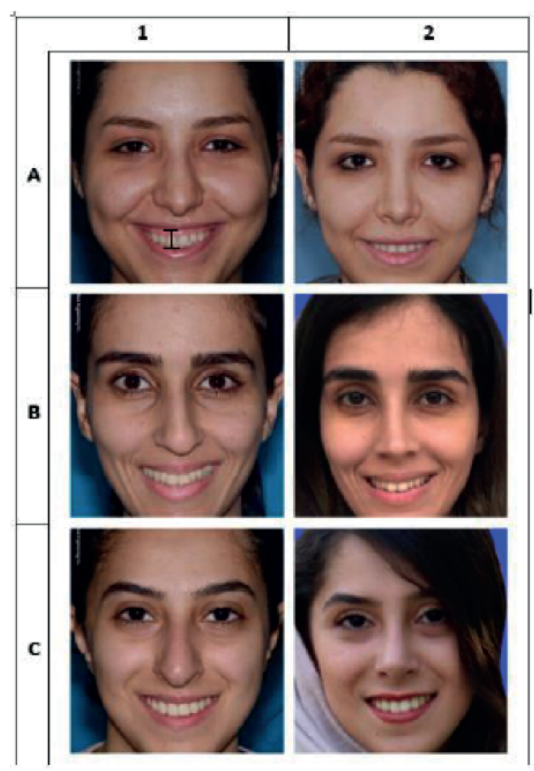

\begin{tabular}{|lcccc|}
\hline MIS change & Number & Mean pre-MIS & Mean post-MIS & p-value \\
\hline Decreased & 9 & $6.56 \pm 1.88$ & $5.56 \pm 1.88$ & .003 \\
\hline No change & 23 & $6.09 \pm 1.44$ & $6.09 \pm 1.44$ & 1.00 \\
\hline Increased & 58 & $5.69 \pm 1.59$ & $7.03 \pm 1.56$ & $<.001$ \\
\hline Total & 90 & $5.88 \pm 1.59$ & $6.64 \pm 1.64$ & $<.001$ \\
\hline
\end{tabular}

Figure 2. The pre- and post-MIS change is presented in the separate table under the figure. The distance, measured as the maxillary incisure show (MIS) ( A, 1). A) Preoperative (column 1) and postoperative (column 2) MIS change photographs of a 31-years old patient with decreased MIS change at smilling. B) Preoperative (column 1) and postoperative (column 2) photographs of a 28-years old patient without MIS change at smilling. C) Preoperative (column 1) and postoperative (column 2) photographs of a 25 -years old patient with increased MIS change at smilling.

\section{Authorship contribution}

Conception or design of the work: AM, MR, FDF, MDF. Drafting the article: AM, FDF, MDF, MR. Data analysis and interpretation: FDF, MDF. Critical revision of the article: AM

All the authors approved the final version and have the agreement to be accountable for all aspects of the work in ensuring that questions related to the accuracy or integrity of any part of the work are appropriately investigated and resolved.

\section{Acknowledgement}

This research did not receive any specific grant from funding agencies in the public, commercial, or not-for-profit sectors. 


\section{References}

1. Batwa W, Grewal B, Gill D. Smile analysis: what to measure. Dent Update. 2014;41(6):483-6, 8-9.

2. Pi H, Kurlander DE, Guyuron B. Effects of the Rhinoplasty Maneuvers on Upper Lip Position and Incisor Show. Aesthetic plastic surgery. 2017:41(1):135-9.

3. Kridel RW, Scott BA, Foda HM. The tonguein-groove technique in septorhinoplasty. A 10-year experience. Arch Facial Plast Surg. 1999;1 (4):246-56; discussion 57-8.

4. Williams EF. Alar-columellar disharmony using the tongue-in-groove maneuver in primary endonasal rhinoplasty. Arch Facial Plast Surg. 2012;14(4):283-8.

5. Toriumi DM, Checcone MA. New concepts in nasal tip contouring. Facial Plast Surg Clin North Am. 2009;17(1):55-90, vi.

6. Honrado CP, Pearlman SJ. Surgical treat- ment of the nasolabial angle in balanced rhinoplasty. Arch Facial Plast Surg. 2003;5(4):338-44.

7. Cerrati EW, Dayan SH. Association of Increasing Nasal Tip Projection With Lip Position in Primary Rhinoplasty. JAMA facial plastic surgery. 2017;19(4):323-6.

8. Kalantar-Hormozi A, Beiraghi-Toosi A. Smile analysis in rhinoplasty: a randomized study for comparing resection and transposition of the depressor septi nasi muscle. Plast Reconstr Surgery. 2014;133(2):261-8.

9. JG McCarthy, Wood-Smith. D. Rhinoplasty. In: McCarthy JG, ed. Plastic Surgery. 1st ed. Philadelphia: Saunders1990.

10. RJ Rohrich, B Huynh, AR Muzaffar, WP Jr Adams, Robinson JJ. Importance of the depressor septi nasi muscle in rhinoplasty: Anatomic study and clinical application. Plast Reconstr Surgery. 2000;105:376-83.

\section{Maryam Roomiani}

ENT and Head \& Neck Research center and department

The five senses Institute Iran University of Medical Sciences Tehran

Iran

Tel: +2122075005

E-mail:

maryamroomiani3@gmail.com

\section{APPENDIX}
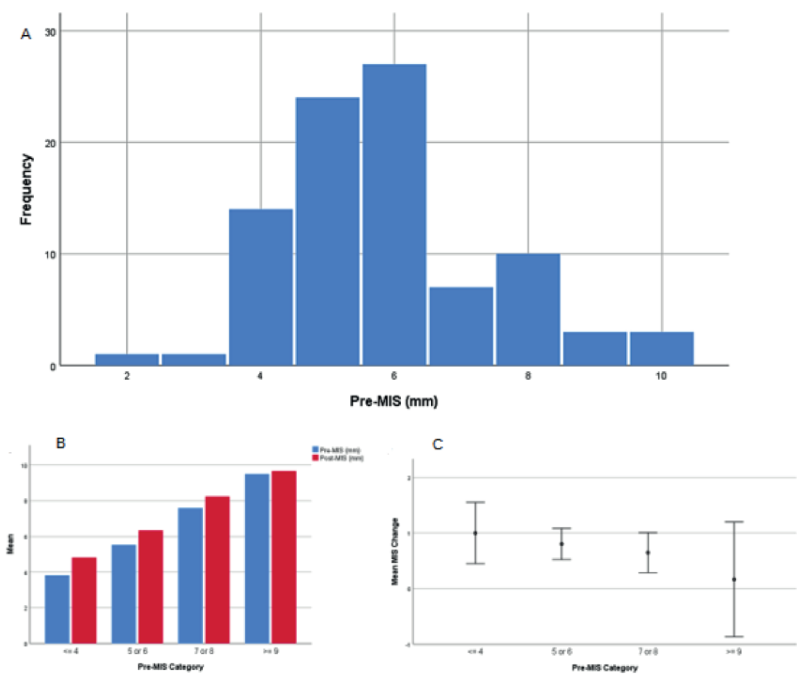

Appendix 1. A) The histogram of the patients' MIS length, before surgery.

$\mathrm{B}, \mathrm{C})$ MIS change among patients with initial MIS $<=4 \mathrm{~mm}, 5$ to 6,7 to 8 ,

and $>=9 \mathrm{~mm}$. a) Pre- vs post-MIS length. b) Mean MIS change. 